\title{
A Wideband High Flat Gain Waveguide-Fed Aperture Antenna Using Superstrate and Shield
}

\author{
Mehdi Ghorbani ${ }^{1}$, Mohsen Khalily ${ }^{2}$, Pei Xiao ${ }^{2}$, Rahim Tafazolli ${ }^{2}$ \\ ${ }^{1}$ Deptartment of electrical engineering, University of Guilan, Rasht, Iran \\ ${ }^{2}$ Institute for Communication Systems (ICS), Home of the $5 G$ innovation center (5GIC), University of Surrey, U.K. \\ ghorbani.me@gmail.com
}

\begin{abstract}
In this paper, a high flat gain waveguide-fed aperture antenna has been proposed. For this purpose, two layers of FR4 dielectric as superstrates have been located in front of the aperture to enhance the bandwidth and the gain of the antenna. Moreover, a conductive shield, which is connected to the edges of the ground plane and surrounding aperture and superstrates, applied to the proposed structure to improve its radiation characteristics. The proposed antenna has been simulated with HFSS and optimized with parametric study and the following results have been obtained. The maximum gain of 13.0 $\mathrm{dBi}$ and $0.5-\mathrm{dBi}$ gain bandwidth of $25.9 \%(8.96-11.63$ GHz) has been achieved. The 3-dBi gain bandwidth of the proposed antenna is $40.7 \%(8.07-12.20 \mathrm{GHz})$, which has a suitable reflection coefficient $(\leqslant-10 \mathrm{dBi})$ in whole bandwidth. This antenna comprises a compact size of $(1.5 \lambda \times 1.5 \lambda)$, easy structure and low-cost fabrication.
\end{abstract}

Keywords-High Flat Gain, Aperture Antenna, Superstrate, Shield, Wideband

\section{INTRODUCTION}

Waveguide antennas have good features, but relatively low gain. There are some techniques which can improve antenna gain including an array of antenna elements $[1,2]$. Another technique, which has been utilized in this paper, is Partially Reflective Surface (PRS or superstrate). This fact was introduced in 1956 by Trentini [3] for the first time and then followed by many works of literatures, including unprinted dielectric [4] or printed dielectric [5], or completely metallic structure [6]. In this technique, using two or three layers of superstrates [7] can enhance antenna gain more efficiently.

For unprinted dielectric superstrates, the maximum gain depends on the permittivity of the dielectric. Therefore, for higher peak gain, using high permittivity dielectric is required. These high permittivity dielectrics increase losses and fabrication cost. Printed dielectric superstrate are more efficient for optimization, leading to achieving higher peak gain, but it often decreases $3-\mathrm{dBi}$ gain bandwidth and disturbs the reflection coefficient. The peak gain, in this technique, follows the hill-shape trend, increasing to maximum level then decreasing to lower values. In wideband application, it can significantly be dropped to lower than peak gain in the side frequencies. In this paper, a new structure is proposed to flatten the antenna gain in a wide range of frequency.

Flat gain concurrently with high gain features in a wideband antenna is favorable subject and was followed in many works of literatures on a variety type of antennas, including microstrip antenna, slot antenna, Yagi antenna etc. In [8], a microstrip patch antenna using a single layer of metamaterial superstrate with a zero refractive index was investigated. A microstrip patch antenna with the radome consist of a pair of parallel strips etched on the bottom of the dielectric [9] realized flat gain feature, but with low peak gain. In [10], a two-pole Butterworth bandpass filter was designed. Then fan shape patch antenna as a second resonator, filtering antenna applied to enhance the gain flatness compared to the traditional antennas.

In [11] square-ring-loaded slot antenna was investigated, which was fed with L-shape microstrip line. In [12], a slot antenna is proposed. In this design, a parasitic patch and windowed superstrate as a gain enhancement method are applied. A planar double-dipole quasi-Yagi antenna using multiple directors as a method of flat gain enhancement was reported in [13]. In [14] printed frequency scanning antenna based on an even-mode bilateral broadside-coupled suspended microstrip line was investigated.

In this paper, a compact size, wideband, and high flat gain antenna has been proposed. The proposed antenna possesses the maximum gain of $13.0 \mathrm{~dB}, 0.5-\mathrm{dBi}$ gain bandwidth of $25.9 \%, 3-\mathrm{dBi}$ gain bandwidth of $40.7 \%$, and good Reflection coefficient at the whole bandwidth. This antenna composes of an aperture mounted on a conducting ground plane, which is fed with the WR90 waveguide. A conducting shield is connected to the edge of the ground plane. Two layers of common and low-cost FR4 dielectric as superstrate are located in front of the aperture and parallel to the ground plane at the distance of $\lambda / 6$ and $\lambda / 2$ approximately. This antenna represents an easy structure and low-cost fabrication, with good radiation characteristics and impedance bandwidth.

\section{ANTENNA CONFIGURATION}

Figure 1 shows the structure of the proposed antenna. The proposed antenna is based on a rectangular aperture mounted on a conductive ground plane. Two layers of FR4 dielectric slabs are located in front of aperture and parallel to the ground plane at a certain distance. Moreover, a shield, which is connected to the ground plane edges, surrounds aperture and superstrates. The excitation is through a waveguide, WR90, with dimensions of $10.16 \times 22.86 \mathrm{~mm}$, and superstrates are FR4 dielectric with $1.6 \mathrm{~mm}$ thickness. 


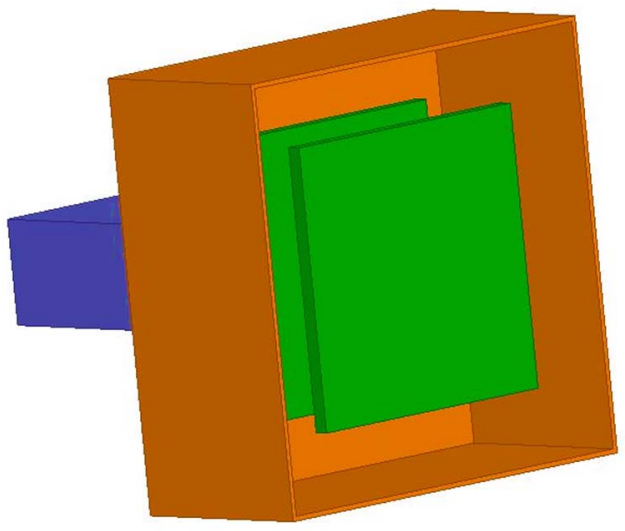

(A)

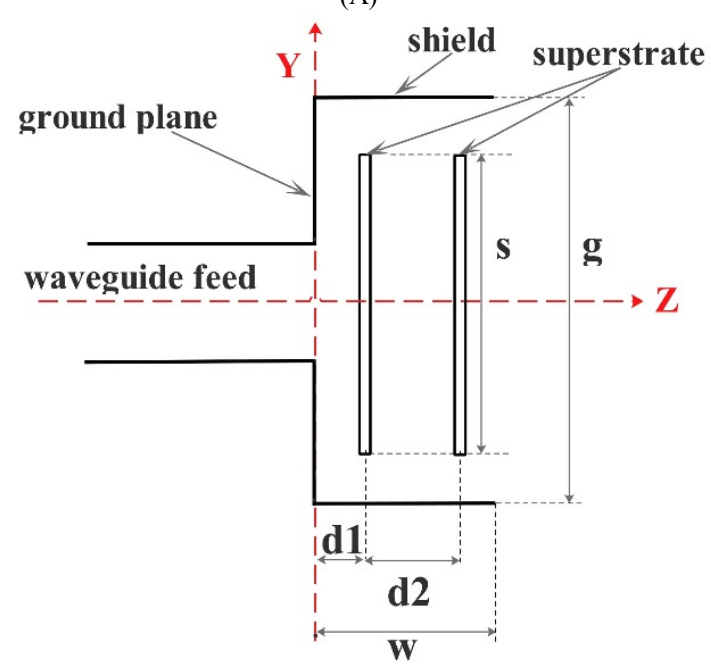

(B)

Fig. 1. Structure of the proposed antenna (A) 3D view (B) side view

The central frequency of the proposed antenna is $10 \mathrm{GHz}$, corresponding to the wavelength of $30 \mathrm{~mm}$. the proposed antenna consists an aperture with dimensions of $22.86 \times 10.16$ $\mathrm{mm}$ (dimensions of WR90) is mounted in the center of conducting ground plane with dimensions of $45 \times 45 \mathrm{~mm}$ $(1.5 \lambda \times 1.5 \lambda)$. Two layers of superstrate slabs with same dimensions of $30 \times 30 \mathrm{~mm}^{2}$ are symmetrically located in front of the aperture at the distances of $5 \mathrm{~mm}(0.17 \lambda)$, and $18 \mathrm{~mm}$ $(0.6 \lambda)$ from the aperture, respectively. Superstrates are chosen common and inexpensive FR4 dielectric with a thickness of $1.6 \mathrm{~mm}$. At the edges of the ground plane, a shield with the profile of the ground plane and width of $22 \mathrm{~mm}(0.73 \lambda)$ is applied to the antenna structure.

\section{ANALYSIS AND PHYSICAL INSIGHT}

In RCAs, a cavity is formed between a superstrate and a ground plane, which resonances at the distance of halfwavelength. The most common method to explore these antennas resonance in accordance with the distance is ray analysis.

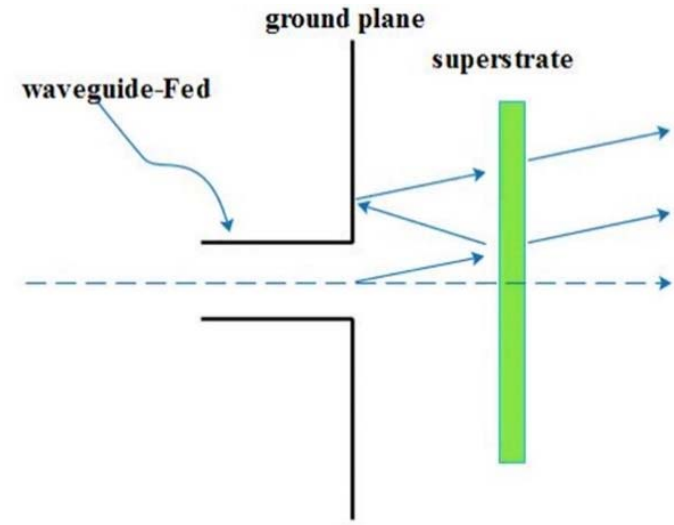

Fig. 2. Resonance cavity antenna superstrate

Fig. 2 shows the resonant cavity antenna which is formed between these two planes. In this way, the distance between superstrate and ground plane is as follows [13]:

$$
s_{d}=\left(\frac{\psi_{0}}{2 \pi}-0.5\right) \frac{\lambda}{2}+N \frac{\lambda}{2}
$$

Where $s_{d}$ is the cavity height, $\psi_{0}$ is the angle of the reflection coefficient, $\lambda$ is the wavelength of central frequency, and $\mathrm{N}$ is a number. To calculate the gain and the bandwidth of an RCA, the following equations are used.

$$
\begin{aligned}
& G=\frac{1+\rho}{1-\rho} \\
& B W=\left(\frac{\lambda}{2 \pi s_{d}}\right) \frac{1-\rho}{\rho^{0.5}}
\end{aligned}
$$

Where $\mathrm{G}$ is the peak gain, $\mathrm{BW}$ is the bandwidth, and $\rho$ is the magnitude of the reflection coefficient. Based on the equation (2), the maximum gain is realized, where the magnitude of reflection coefficient $\rho=1$ for highly reflective FSS. Therefore, $\psi_{0}=\pi$ is achieved and for $\mathrm{N}=1, \mathrm{~s}_{\mathrm{d}}=\lambda 2$.

\section{DESIGN AND SIMULATION}

\section{A. Design steps}

In the proposed antenna, there are 5 critical parameters of $\mathrm{s}, \mathrm{g}, \mathrm{w}, \mathrm{d}_{1}$, and $\mathrm{d}_{2}$. The main target of this paper is to design wideband and high flat gain. Therefore, the reflection coefficient and gain chart of the proposed antenna with these parameters is investigated.

Step 1: At the first step, ground plane dimensions is studying. For this purpose, the ground plane is assumed in a square shape with dimensions of $g \times g$. Fig. 3(A) and (B) depict the gain and Reflection coefficient of the proposed antenna in accordance with the parameter of $g$, respectively. 


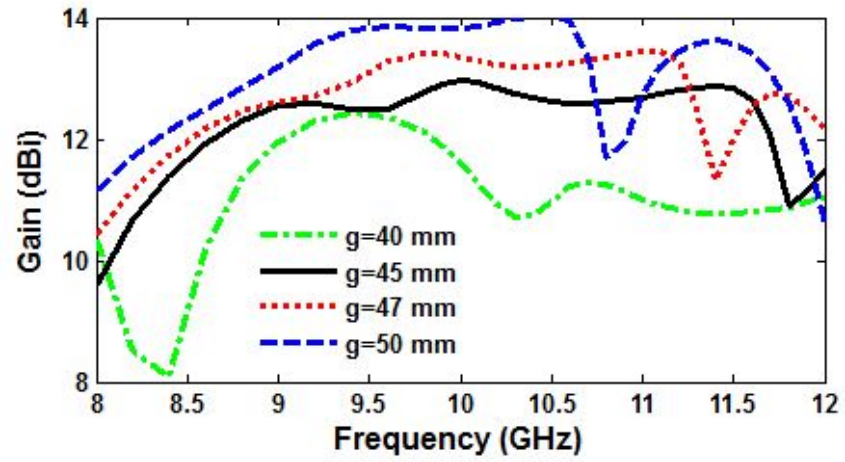

(A)

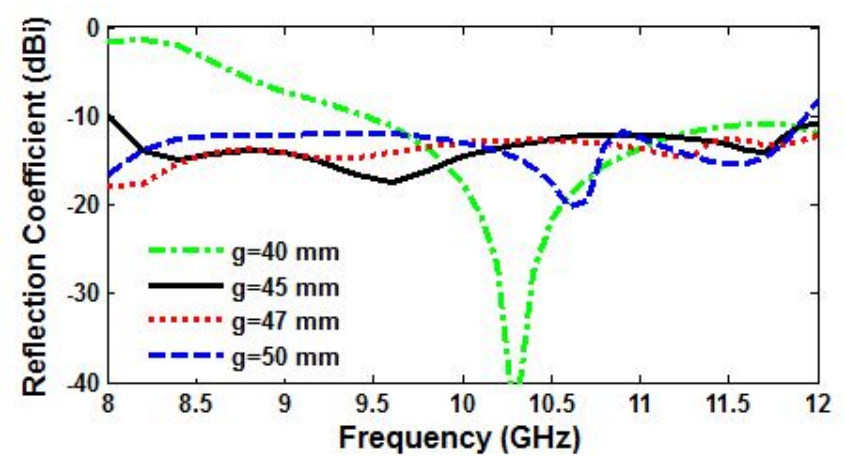

(B)

Fig. 3. (A) Gain and (B) Reflection coefficient of the proposed antenna with the parameter of $\mathrm{g}$ (ground plane dimensions)

Fig. 3(A) shows that higher values of $g$ enhance the maximum gain, but the gain variation increases. Fig 3(B) shows that $\mathrm{g}=45 \mathrm{~mm}$ is the best choice for the ground plane dimensions.

Step 2: At the second step, superstrates dimensions are investigated. Similar to the ground plane, the superstrates assume a square shape with the dimensions of $\mathrm{s}^{\times} \mathrm{s}$. Fig. 4(A) and 4(B) respectively show the gain and the Reflection coefficient of the proposed antenna in accordance with the parameter of s. Fig. 4(A) shows that the gain of the antenna at lower frequencies is approximately unchanged with the variation of the parameter of $p$, and at higher frequencies, it is increased while parameter of $s$ is decreased. Also, figure 4(B) shows that increasing of the superstrate size improves the Reflection coefficient of the proposed antenna. Therefore, $\mathrm{s}=30 \mathrm{~mm}$ is the best choice for the flat gain feature.

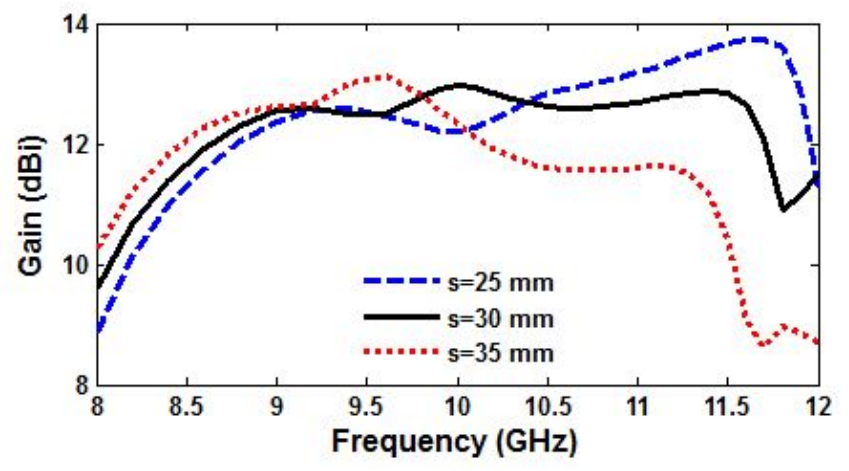

(A)

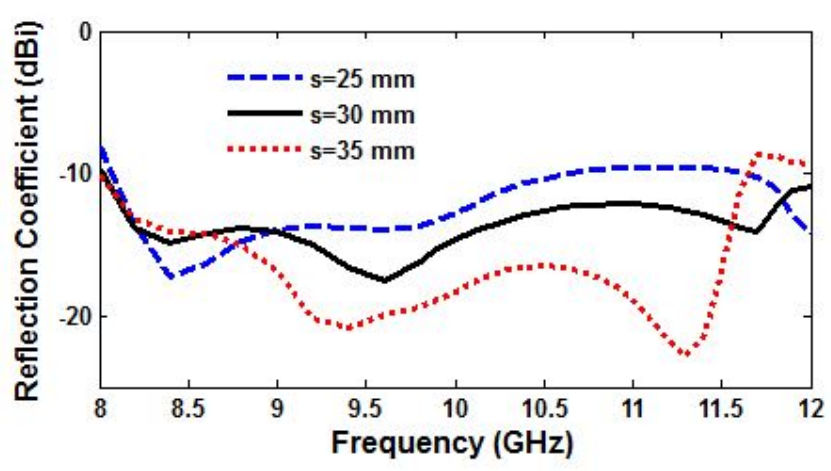

(B)

Fig. 4. (A) Gain (B) Reflection coefficient of the proposed antenna with the parameter of s (superstrate dimensions)

Step 3: Fig. 5(A) and (B) show the gain and Reflection coefficient of the proposed antenna with the conducting shield length, w. Figures 5(A) and 5(B) show that the parameter of $\mathrm{W}$ changes the gain at the middle frequencies, and the gain is approximately steady at the lower and the higher frequencies. According to these figures, $\mathrm{w}=22 \mathrm{~mm}$ selected as the best value for the conducting shield length.

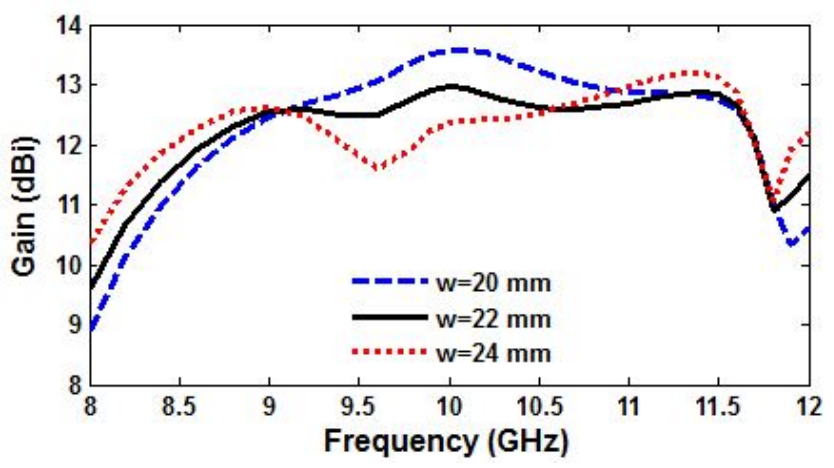

(A)

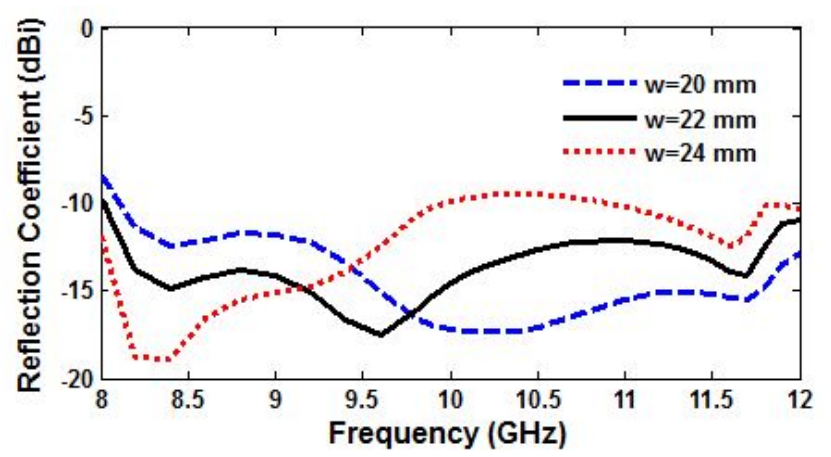

(B)

Fig. 5. (A) Gain (B) Reflection coefficient of the proposed antenna with the parameter of w (shield length)

Step 4: The distance between the first superstrate and ground plane $\left(\mathrm{d}_{1}\right)$ is the next critical parameter that must be investigated and optimized. Figure 6(A) and 6(B) depict the gain and the Reflection coefficient of the proposed antenna in accordance with $d_{1}$ variations, respectively. It can be seen that $\mathrm{d}_{1}=5 \mathrm{~mm}$ is the best value for this parameter. 


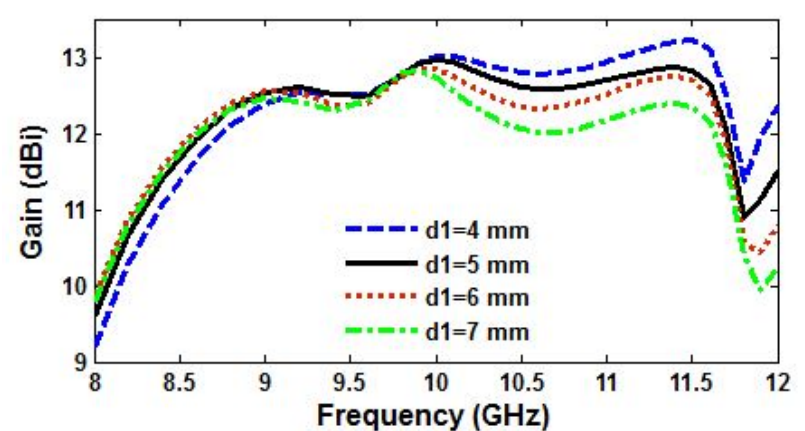

(A)

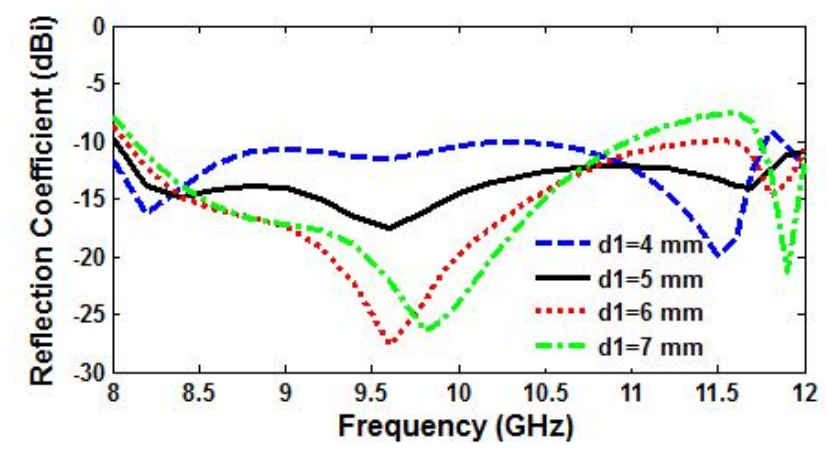

(B)

Fig. 6. (A) Gain (B) Reflection coefficient of the proposed antenna with the parameter of $d_{1}$

Step 5: At the final step, the distance between the second superstrate from the ground plane, $\mathrm{d}_{2}$, is investigated. Fig. 7(A) and (B) respectively show the gain and the Reflection coefficient of the proposed antenna in accordance with $\mathrm{d}_{2}$. According to these charts, $\mathrm{d}_{2}=18 \mathrm{~mm}$ is the best choice of optimization.

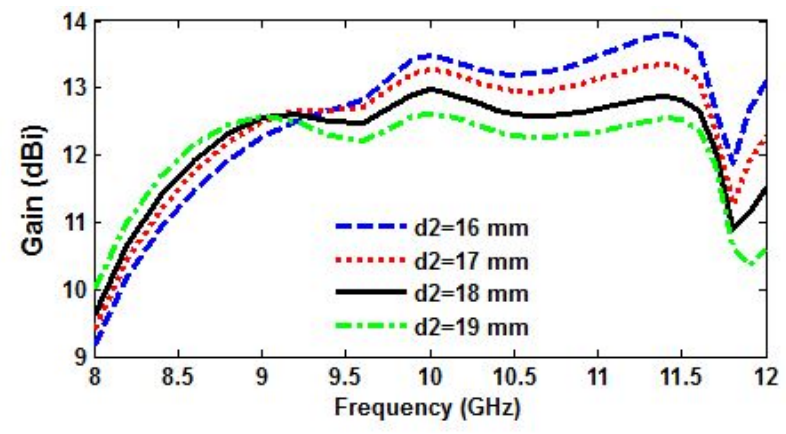

(A)

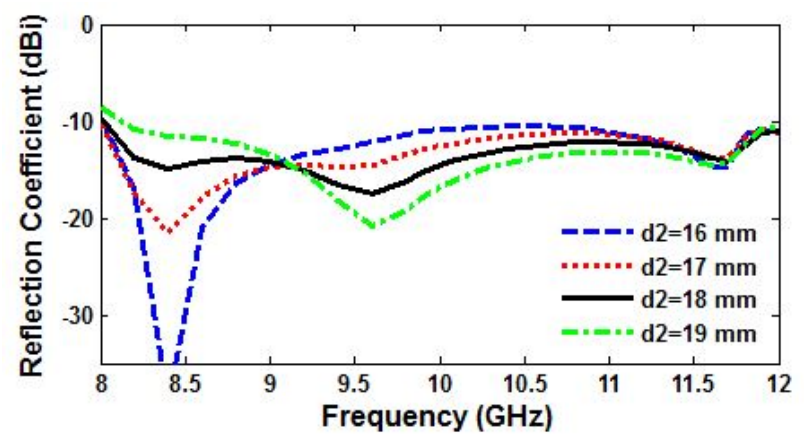

(B)

Fig. 7. (A) Gain (B) Reflection coefficient of the proposed antenna with the parameter of $d 2$
Fig. 8(A) and (B) show the final results of the gain and the Reflection coefficient of the proposed antenna compared with the final structure without the shield, and the final structure without superstrates.

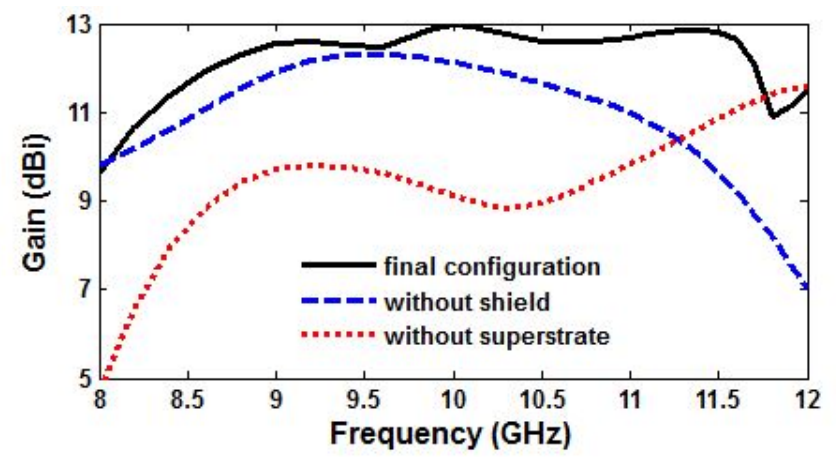

(A)

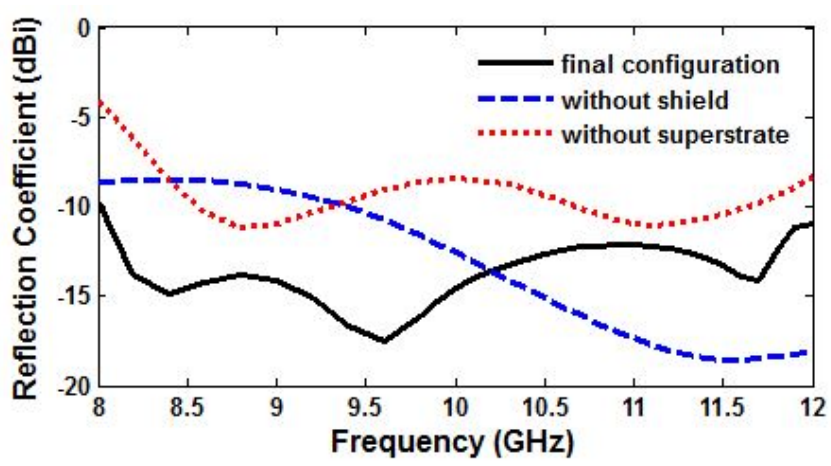

(B)

Fig. 8. (A) Gain (B) Reflection coefficient of the final configuration of the proposed antenna compared to the only superstrates and only shield

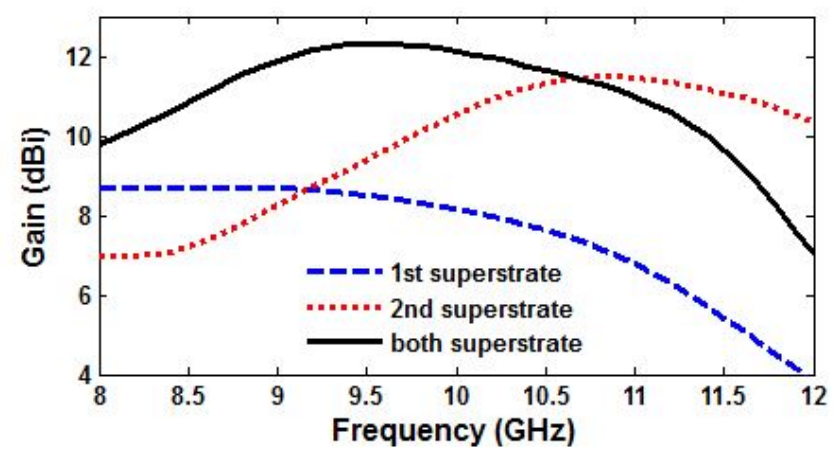

(A)

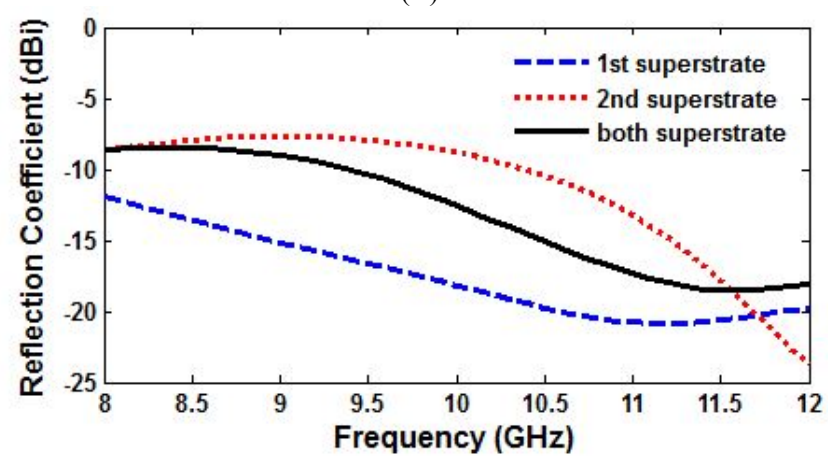

(B)

Fig. 9. (A) Gain (B) of final configuration of the proposed antenna compared to the only superstrates and only shield 
Fig. 9(A) and (B) show the gain and the Reflection coefficient of the proposed antenna in the case of without shield and only first superstrate alone, only second superstrate, and both superstrates concurrently. According to these figures, the case of only first superstrate has a good Reflection coefficient but weak gain, particularly at higher frequencies. In the case of only second superstrate, it has a weak Reflection coefficient and good gain at the higher frequencies. This antenna with both superstrates together and without shield has a gain of Hill-shape. This antenna with shield and without superstrates have a good gain at higher frequencies, but weak Reflection coefficient in the whole bandwidth. Combination of these two superstrates and the shield create a good structure with high flat gain at a wide range of frequency with good Reflection coefficient in the whole bandwidth.

\section{B. Simulation Results}

The proposed antenna has a maximum gain of $13.0 \mathrm{dBi}$ at the central frequency of $10 \mathrm{GHz}$. This antenna has an approximately flat gain at $2.67 \mathrm{GHz}(8.96-11.63 \mathrm{GHz})$, which the minimum gain in this bandwidth is $12.5 \mathrm{~dB}$. Also, an excellent $3-\mathrm{dBi}$ gain bandwidth of $40.7 \%$ is achieved, with a good Reflection coefficient in the whole bandwidth. In this structure, just two layers of common and low-cost FR4 dielectric are used, and a compact size antenna is realized with the easy and inexpensive structure to fabricate. Figure 10 shows the radiation patterns of E-plane and H-plane at the central frequency. The side lobe and back lobe of the proposed antenna at the central frequency is $18.1 \mathrm{dBi}$ and $20.2 \mathrm{~dB}$, respectively.

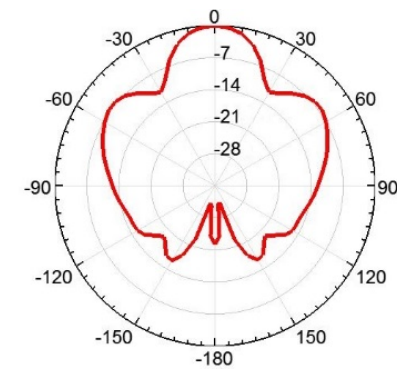

(A)

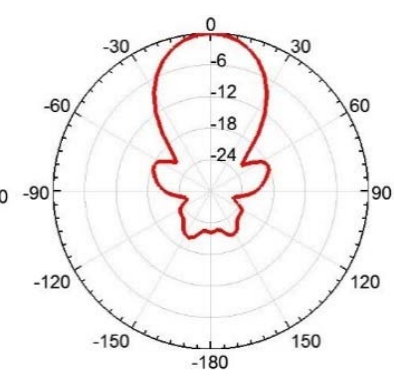

(B)
Fig. 10. (A) E-plane (B) H-plane of the proposed antenna at $10 \mathrm{GHz}$

\section{CONCLUSION}

In this paper, a waveguide fed aperture antenna with a shield and two layers of superstrates has been proposed. The proposed antenna composed of an aperture mounted on an extended conducting ground plane, together with two layers of FR4 dielectrics as a superstrate, which is located in front of the aperture at the distance of approximately $\lambda / 6$ and $\lambda / 2$, and a conducting shield, which is connected to the edges of the ground plane and surround superstrates. This structure creates a flat gain antenna at a wide range of frequency, and also appropriate Reflection coefficient at all of the bandwidth. The dimension of the ground plane, two superstrates, and shield is investigated and optimized using HFSS. The antenna outputs include the maximum gain of $13.0 \mathrm{~dB}, 0.5-\mathrm{dBi}$ gain bandwidth of $25.9 \%$, and $3-\mathrm{dBi}$ gain bandwidth of $40.7 \%$. The reflection coefficient of the proposed antenna is less than $-10 \mathrm{dBi}$ at the whole bandwidth. Moreover, this antenna has a compact size of $1.5 \lambda \times 1.5 \lambda$, with simple and inexpensive structure and material. The proposed antenna also have a suitable side lobe and back lobe.

\section{REFERENCES}

[1] N.M. Nor, M.H. Jamaluddin, M.R. Kamarudin, M. Khalily, "Rectangular dielectric resonator antenna array for $28 \mathrm{GHz}$ applications", Progress In Electromagnetics Research 63, 53-61

[2] M. Khalily, R. Tafazolli, P. Xiao, A.A. Kishk, "Broadband mm-Wave Microstrip Array Antenna with Improved Radiation Characteristics for Different 5G Applications", IEEE Transactions on Antennas and Propagation, 2018, Vol. 66, Iss. 9, pp 4641 - 4647

[3] G.V. Trentini, "Partially reflecting sheet array", Transaction on Antennas and Propagation,Vol.4, 1956, pp.666-671

[4] B.A. Zeb, R.M. Hashmi, K.P. Esselle, "Wideband gain enhancement of slot antenna using one unprinted dielectric superstrate", ELECTRONICS LETTERS, 2015, Vol. 51, No. 15, pp. 1146-1148

[5] M. Ghorbani, H. Ghorbaninejad, "Design of a High Gain Bandwidth Improved Aperture Antenna Using a Frequency Selective Surface", ACES JOURNAL, Vol. 32, No.4, April 2017

[6] R. Fakhte, H. Ghorbaninejad, " High gain and improved waveguide slot antenna using a metallic superstrate as main radiator", IET Microwaves, Antennas \& Propagation 11 (4), 2016, pp. 557-563

[7] M. Ghorbani, H. Ghorbaninejad, "Novel High-Gain Narrowband Waveguide-Fed Filtenna using Genetic Algorithm", International Journal of Electrical and Computer Engineering (IJECE), Vol. 7, No. 1, February 2017, pp. 253-259

[8] J. Ju, D. Kim, W.J. Lee, J.I. Choi, "Wideband High-gain Antenna Using Metamaterial Superstrate with the Zero Refractive Index", Microwave And Optical Technology Letters, Vol. 51, No. 8, August 2009

[9] T.N. Chang, J.M. Lin, "Enhanced Return-Loss and Flat-Gain Bandwidths for Microstrip Patch Antenna", IEEE Transactions on Antennas and Propagation, Vol. 59, No. 11, November 2011

[10] X. Chen, F. Zhao, L. Yan., W. Zhang, "A Compact Filtering Antenna with Flat Gain Response within the Passband", IEEE Antennas and Wireless Propagation Letters, Vol. 12, 2013

[11] H. Zhang, Y.C. Jiao, L. Lu, and C. Zhang, "Broadband Circularly Polarized Square-Ring-Loaded Slot Antenna With Flat Gains", IEEE Antennas and Wireless Propagation Letters, Iss. 99, 2016

[12] Z.H. Tu, Q.X. Chu, Q.Y Zhang, "High-Gain Slot Antenna with Parasitic Patch and Windowed Metallic Superstrate", Progress In Electromagnetics Research Letters, Vol. 15, pp. 27-36, 2010

[13] J. Yeo, J.I. Lee, "Broadband Flat Gain Enhancement of Planar DoubleDipole Quasi-Yagi Antenna Using Multiple Directors", Progress In Electromagnetics Research C, Vol. 65, pp. 1-9, 2016

[14] L. Cui, W. Wu, D.G. Fang, "Printed Frequency Beam-Scanning Antenna With Flat Gain and Low Sidelobe Levels", IEEE Antennas And Wireless Propagation Letters, Vol. 12, 2013 